\section{Frequency Drift of Short-Time Transients in Solar Radio Noise}

A DETAILED knowledge of the structure of solar noise storm bursts is essential for a theoretical treatment and understanding of the phenomenon. Different methods of observation have been tried in order to secure information on important physical quantities of storm bursts. In this communication some preliminary results are reported from an analysis of highspeed records which were taken with a receiver containing two channels centred at $199 \mathrm{Mc}$./sec. and $200.5 \mathrm{Mc} /$ sec. (band-width : $0.3 \mathrm{Mc} / \mathrm{sec}$., time constant 0.01 sec., and noise factor about 4). Records were taken with a double-channel Brush recorder at paper-speeds of 5, 25 and $125 \mathrm{~mm}$./sec. Records have been obtained since February of this year during most noise storm periods.

A first inspection of the records gives the impression that the run of intensity versus time on the two frequencies often shows poor correspondence. This is in agreement with earlier observations, which have shown that storm bursts are of a highly monochromatic character. A more surprising feature, however, is the frequently occurring time difference between corresponding pips in the channels. Some good examples are shown in Fig. 1, $a, b, c$. In Fig. 1, $a$ the low-frequency signal is in advance of that on the higher frequency, but in Fig. 1,b, the opposite is the case, while in Fig. 1,c the pips occur simultaneously. During a noise storm these three possibilities are all realized, but in different proportions. On April 28, out of 87 pairs of pips, 42 occurred at first on the low frequency, 29 occurred first on the high frequency, and 16 occurred simultaneously.

The simplest way to explain the observations is to suppose that the transients in most cases have a
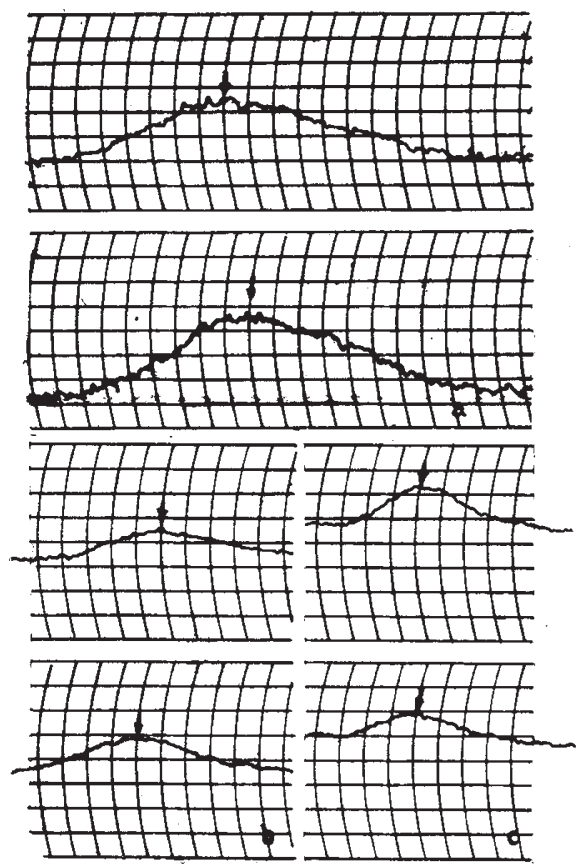

Fig. 1. Examples of records. (Paper speed $125 \mathrm{~mm} . / \mathrm{sec} .$, lowerfrequency above.) In $(a)$ the pip occurs first on the low frequency,
in $(b)$ on the high frequency and in $(c)$ simultaneously in both
channels frequency drift from higher to lower frequencies, or vice versa. If this is assumed, observations indicate drift velocities in either direction ranging from about $10 \mathrm{Mc} . / \mathrm{sec}^{2}$ and upwards to rather high values.

Observations by Wild ${ }^{1}$ with a spectrometer covering the frequency range $70-130 \mathrm{Mc}$./sec. showed that noise storm bursts had a mid-frequency which remained approximately constant throughout their life-time. However, since the rather extended spectral range covered was only displayed about three times a second, the instrument was not fitted for observations of transients with a duration less than, say, $0.5 \mathrm{sec}$. and a drift over only a few megacycles/second. This may be the reason why the effect dealt with here has not been reported earlier. The mean duration of the pips which were recorded with the twochannel receiver was of the order of $0.30 \mathrm{sec}$. A narrow-band spectrometer with a high frequency of repetition has now been erected at the Solar Observatory.

A fuller analysis of the records from the doublechannel receiver is in preparation and the results will soon be published.

This investigation is being supported in part by the U.S. Air Force (ARDC, European Office).

\section{ÖYSTEIN ElgaRöy}

Solar Observatory,

Institute of Theoretical Astrophysics, University of Oslo.

Sept. 14.

${ }^{1}$ Wild, J. P., Austral. J. Sci. Res., 4, (1), 36 (1951).

\section{Strength of Highly Porous Brittle Materials}

THE experimental scatter in strength measurements of brittle materials is now accepted as reflecting the inherent statistical nature of this type of fallure rather than an excessive sensitivity to measuring methods. Moreover, some characteristic features of brittle bodies, such as the scale effect, whereby geometrically similar test pieces of different absolute size give significantly different results, are easily explained on a statistical basis.

The fundamental work on these questions has been done by W. Weibull and by J. I. Frenkel and T. A. Kontorova, who base their theory on a random distribution of microscopic flaws in the material. These flaws weaken the material in their vicinity to different extents, the most 'dangerous' flaw determining the macroscopic strength of a homogeneously stressed test piece. At the back of these ideas is the old theory of 'Griffith's cracks', which are the cause of local stress concentrations, the severity of which depends on the size of crack.

These theories are satisfactory if they are to explain only the statistical nature of brittle fracture. The notion of a flaw, however, is too vague to give a clear-cut relation between the inherent strength of the material and the weakening effect of these flaws. The Griffith's crack, it is true, does give such a relation, but it forms a very special type of flaw; the number of such cracks per unit volume of the material is, moreover, usually assumed to be so low that the stress concentration in the neighbourhood of one is not affected by the presence of another crack. Neither the shape nor the concentration of 